\title{
Fatores Associados à Ocorrência da Síndrome de Burnout entre Estudantes de Residências Multiprofissionais
}

\section{Factors Associated with Burnout Syndrome in Multiprofessional Residents}

\author{
Rosângela Fernandes de Oliveira ${ }^{I}$ (iC) \\ Maria Amélia Dias Pereira ${ }^{I}$ D \\ Matheus Lopes da Silva ${ }^{I}$ (D) \\ Matheus Leão Tavares Costa ${ }^{I}$ (ID \\ Érika Carvalho Quirino ${ }^{I}(\mathbb{D})$ \\ Alessandra Vitorino Naghettini ${ }^{I}$ iD
}

\section{PALAVRAS-CHAVE}

- Residência.

- Burnout.

- Ensino.

\section{RESUMO}

Introdução: Os residentes multiprofissionais da saúde lidam diariamente com alto nível de estresse, favorecendo o prejuízo ao atendimento do paciente, o que pode causar reações emocionais e interpessoais que contribuem para o desenvolvimento da síndrome de burnout, revelando, assim, a importância de identificar as condições de risco para a síndrome e a possível proteção. O objetivo deste estudo foi descrever a ocorrência de burnout, suas dimensões de comprometimento e a associação com características sociodemográficas entre profissionais da residência multiprofissional. Método: Trata-se de uma pesquisa descritiva, com abordagem quantitativa, realizada com residentes multiprofissionais em saúde de hospitais públicos localizados em uma capital do CentroOeste do Brasil. Adotou-se o Inventário da Síndrome de Burnout (ISB), utilizando-se de seis índices (condições organizacionais positivas, condições organizacionais negativas, exaustão emocional, distanciamento emocional, desumanização e realização profissional), associados à avaliação de características sociodemográficas, como sexo, estado civil, profissão, trabalho anterior, mudança de cidade, morar sozinho, ter filhos, fazer outro curso, gostar do que faz nas horas vagas, praticar atividade física, ter religião, estar em processo terapêutico. O método de regressão logística foi utilizado para verificar a associação entre essas características e o diagnóstico da síndrome de burnout. Participaram da pesquisa 134 residentes. Resultados: Evidenciou-se que a exaustão emocional está presente em $91 \%$ dos participantes; o distanciamento emocional em 89,6\%; a desumanização em 61,9\%; a realização profissional em 11,2\%; as condições organizacionais positivas em $85,1 \%$; e as condições organizacionais negativas em $82,1 \%$. A associação positiva foi feita entre a realização de cursos simultâneos, a característica sexo masculino e o fato de morar sozinho. Conclusóes: Observa-se que a prática de terapia pode reduzir a chance do desenvolvimento da síndrome e conclui-se que há uma alta prevalência da síndrome de burnout no grupo pesquisado, sendo resposta ao estresse definido pela presença de exaustão, distanciamento e desumanização. Apesar desses resultados, a percepção de realização profissional se mantém independente do estresse. 


\section{KEYWORDS}

- Residence.

- Burnout.

- Teaching.

\section{ABSTRACT}

Introduction: Multiprofessional health residents deal with high stress daily, harming patient care, which can cause emotional and interpersonal reactions that contribute to the development of Burnout Syndrome, thus disclosing the importance of identifying the at-risk conditions for the syndrome and possible protection. The objectives of this study were to describe the occurrence of burnout, its impairment dimensions and the association with sociodemographic characteristics in multiprofessional residency professionals. Method: This is a descriptive research with a quantitative approach, conducted with multiprofessional health residents from public hospitals located in a capital of the Midwest region of Brazil. The ISB - Burnout Syndrome Inventory - was employed, using six indices (Positive Organizational Conditions, Negative Organizational Conditions, Emotional Exhaustion, Emotional Distancing, Dehumanization and Professional Achievement), associated with the assessment of sociodemographic characteristics such as gender, marital status, profession, previous work, change of city, living alone, having children, getting another degree, enjoying what you do in your free time, practicing physical activity, having a religion, undergoing a therapeutic process. The logistic regression method was used to verify the association between these characteristics and the diagnosis of burnout syndrome. A total of 134 residents participated in the survey. Results: The results showed that Emotional Exhaustion is present in 91\% of participants; Emotional Distance in 89.6\%; Dehumanization, in 61.9\%; Professional Achievement in 11.2\%; Positive Organizational Conditions, in $85.1 \%$; and Negative Organizational Conditions, $n 82.1 \%$. A positive association was observed between taking simultaneous courses, male gender and living alone. Conclusion: It is observed that the practice of therapy can reduce the chance of developing the syndrome and it is concluded that there is a high prevalence of burnout syndrome in the assessed group, with it being a response to stress defined by the presence of exhaustion, distancing and dehumanization. Despite these results, the perception of professional achievement remains independent of stress.

Recebido em 17/12/19

Aceito em 19/2/20

\section{INTRODUÇÃO}

A residência multiprofissional em saúde constitui uma modalidade de ensino de pós-graduação lato sensu destinada às profissões de saúde, sob forma de curso de especialização, com o objetivo de articular e integrar o saber no cuidado à saúde. No exercício das atividades, os residentes deparam-se com uma demanda de difícil controle, como sequelas graves, pacientes com prognósticos muito ruins e morte. Os sentimentos de impotência e culpa são frequentes na rotina de trabalho, o que pode acarretar implicações na saúde, como a síndrome de burnout ${ }^{1}$.

O processo de formação profissional leva os estudantes da residência multiprofissional em saúde a inserir-se em ambientes de trabalho hospitalares, com demandas específicas do meio laboral, cobrança quanto a cumprimento de horários, exposição a materiais de riscos e assumência de responsabilidades pelos usuários, o que os deixa em condições de muita exposição ao estresse e consequentemente ao burnout, podendo levá-los a grande prejuízo no processo de aprendizado ${ }^{2}$.

O termo burnout foi definido por Maslach e Jackson ${ }^{3}$ como uma síndrome de exaustão emocional, despersonalização e redução de realização pessoal, causada pelo prolongamento de situações de elevada exigência emocional no ambiente de trabalho. É a resposta do indivíduo a esse estresse, percebida na mudança da postura em relação às atividades profissionais, como o baixo desempenho das funções, distanciamento e aumento de doenças ${ }^{4}$.

Nesta pesquisa, utilizamos, para a avaliação da síndrome de burnout, o inventário validado pela autora Benevides Pereira ${ }^{5}$ : Inventário da Síndrome de Burnout (ISB). No ISB, há três dimensões que representam os eixos de apresentação da síndrome: exaustão emocional (EE), desumanização (DES) e baixa realização profissional (RP). Esse instrumento avalia as condições operacionais positivas (COP), que promovem o bem-estar e a qualidade de vida, e as condições operacionais negativas (CON), que contribuem para o desencadeamento da síndrome (Tabela 1).

\begin{tabular}{|c|c|c|c|}
\hline \multicolumn{4}{|c|}{$\begin{array}{c}\text { Tabela } 1 \\
\text { Classificação de risco }\end{array}$} \\
\hline \multirow{2}{*}{ Índices } & \multicolumn{3}{|c|}{ Classificação do risco } \\
\hline & Alto risco & Médio risco & Baixo risco \\
\hline Condições organizacionais positivas & Inferior a 22 & Entre 22 e 26 & Superior a 26 \\
\hline Condições organizacionais negativas & Superior a 13 & Entre 8 e 13 & Inferior a 8 \\
\hline Exaustão emocional & Superior a 9 & Entre 4 e 9 & Inferior a 4 \\
\hline Distanciamento emocional & Superior a 6 & Entre 2 e 6 & Inferior a 2 \\
\hline Desumanização & Superior a 7 & Entre 4 e 7 & Inferior a 4 \\
\hline Realização profissional & Inferior a 10 & Entre 10 e 15 & Superior a 15 \\
\hline
\end{tabular}

Fonte: Benevides-Pereira ${ }^{5}$ 
Entender o cenário de ensino-aprendizagem e sua influência no bem-estar desses profissionais em processo de formação é essencial, pois mudanças nos ambientes de trabalho proporcionam correlação com a qualidade de vida e, consequentemente, com a prevalência do burnout ${ }^{6}$.

Este trabalho tem como objetivo identificar a ocorrência da síndrome de burnout entre os residentes da residência multiprofissional em saúde, avaliando as dimensões de comprometimento e sua associação com características sociodemográficas.

\section{METODOLOGIA}

Realizou-se um estudo de caráter transversal, descritivo e quantitativo, com residentes da residência multiprofissional em saúde dos hospitais públicos de uma capital no Centro-Oeste do Brasil. Convidaram-se os 176 residentes que estavam matriculados na residência multiprofissional em saúde, na cidade, no período de 2016 a 2017, dos quais 134 aceitaram participar da pesquisa.

A amostra do estudo foi composta por residentes de ambos os sexos, sendo 80 do primeiro ano (R1) e 54 do segundo ano (R2), de hospitais públicos que oferecem a residência multiprofissional em saúde na capital pesquisada, totalizando 134 participantes.

Os residentes que estavam de férias ou afastados das funções acadêmicas durante o período de coleta de dados foram excluídos da pesquisa.

A coleta dos dados foi realizada entre os meses de agosto e novembro de 2017, após contato prévio com os tutores de cada unidade, nos hospitais onde os residentes faziam as atividades acadêmicas e assistenciais.

Após a explicação dos objetivos do estudo, aqueles que concordaram em participar assinaram o Termo de Consentimento Livre e Esclarecido (TCLE). Os instrumentos de avaliação foram respondidos, de forma individual, pelos residentes, em salas que garantiram a privacidade e o sigilo. Nenhum instrumento foi devolvido de modo incompleto.

Para avaliação sociodemográfica, utilizou-se um questionário elaborado especialmente para esta pesquisa, que analisava as seguintes variáveis: sexo, estado civil, profissão, trabalho anterior, mudança de cidade, morar sozinho, ter filhos, fazer outro curso simultaneamente, gostar do que faz nas horas vagas, praticar atividade física, ter religião e estar em processo terapêutico.

$\mathrm{O}$ instrumento utilizado para avaliar a síndrome de burnout foi o ISB, desenvolvido por Benevides Pereira ${ }^{5}$. Tal instrumento é composto por 35 itens distribuídos em duas partes (16 e 19 itens, respectivamente). A primeira parte avalia os fatores antecedentes ou organizacionais indicados pela literatura como desencadeantes ou moduladores de processos de estresse ocupacional e, consequentemente, burnout, que são divididos em COP e CON. A segunda parte avalia a síndrome por meio de quatro dimensões: EE, distanciamento emocional (DE), DES e realização pessoal $(\mathrm{RP})^{4}$.

Nesse instrumento de avaliação, as dimensões DE e DES são desdobramentos da despersonalização ou do cinismo descritos em outros testes que avaliam burnout. O ISB apenas acrescenta essa divisão.

Os itens estão compostos por afirmações que devem ser respondidas em uma escala Likert de cinco pontos, e, na primeira parte, são escalonadas as respostas de 0 ("nunca" a 4 ("muito frequentemente"), e, na segunda parte, de 0 ("nunca") a 4 ("todos os dias") 4

Esse projeto obteve aprovação no Comitê de Ética e Pesquisa, registrado sob o ${ }^{\circ}$ 70432117.2.0000.5078 e Parecer no 2.209.833.
Análise de dados

Para caracterizar a síndrome de burnout, utilizaram-se seis critérios (COP, CON, EE, DE, DES e RP), cuja pontuação determina o risco a que o participante se encontra exposto. Vale ressaltar que as COP e a RP se correlacionam com a síndrome de maneira inversa às outras variáveis, e maiores números representam riscos menores.

Para caracterizar a presença da síndrome de burnout, a pontuação de um indivíduo avaliado deve indicar altos níveis de pelo menos dois dos seguintes critérios: EE, DE e DES. Essas dimensões são consideradas ponto central da síndrome de burnout, por conterem interações entre elevadas demandas e reduzidos recursos de enfrentamento, ao passo que a dimensão RP se apresenta comprometida posteriormente ${ }^{4}$.

Com o propósito de detalhar a distribuição das pontuações dos indivíduos com relação a cada um dos índices, utilizaram-se medidasresumo (mediana, média, desvio padrão, mínimo e máximo).

O método de regressão logística foi utilizado para verificar a associação entre essas características e o diagnóstico de síndrome de burnout. Construiu-se também um modelo de regressão logística multivariada, em que a variável resposta foi ajustada levando em conta mais de uma variável explicativa (covariável). Inseriram-se, nesse modelo, variáveis cujo $\mathrm{P}$-valor foi menor que 0,2 na análise de regressão logística bivariada.

\section{RESULTADOS}

Em relação ao perfil sociodemográfico dos residentes multiprofissionais, observou-se o predomínio do sexo feminino, $89,55 \%$; solteiros, $81,34 \%$; naturais da cidade onde fazem a residência, $68,66 \%$; não moram sozinhos, $75,37 \%$; e não trabalhavam antes da residência, $71,64 \%$. Apenas $9,7 \%$ dos participantes possuem filhos e mais da metade, $53,73 \%$, não pratica atividade física.

Depois da análise do questionário ISB, observou-se que, entre os 134 residentes entrevistados, $84,33 \%$ foram identificados com a síndrome de burnout.

Após a análise da associação entre os aspetos sociodemográficos e a presença da síndrome de burnout, verificou-se uma predominância de residentes solteiros, $82,3 \%$, que faziam curso concomitante, $20,4 \%$. A análise da associação dos fatores sociodemográficos não identificou diferenças estatisticamente significativas entre residentes que estão em processo terapêutico (Tabela 2).

$\mathrm{Na}$ avaliação da associação pela regressão logística, observou-se então o risco aumentado para aqueles que faziam outro curso simultâneo à residência, para os de sexo masculino, para os que moravam sozinhos e para os que mudaram de cidade, apesar de não se apresentarem como estatisticamente significantes.

A distribuição da frequência de burnout encontrada entre os residentes de acordo com suas respectivas profissões foi similar. A menor frequência da síndrome foi entre psicólogos, $75 \%$, seguida pelos fonoaudiólogos, $80 \%$. Entre os assistentes sociais, $80 \%$ apresentaram, e, entre os enfermeiros, $84,62 \%$. Fisioterapeutas, farmacêuticos e nutricionistas apresentaram, respectivamente, $87,5 \%, 87,5 \%$ e $90 \%$. Terapia ocupacional e biomedicina apresentaram $100 \%$ de ocorrência de burnout, tendo, respectivamente, quatro e seis residentes pesquisados.

$\mathrm{Na}$ análise da dimensão $\mathrm{EE}$, obteve-se o escore médio de 15,48 +- 3,77 , com $91 \%$ dos residentes pertencentes à categoria de alto risco. Quando se avaliou o DE, o escore médio foi de $11.57+-4,58$, com $89 \%$ na categoria de alto risco. Na dimensão DES, o escore médio foi de 8,79 $+-3,88$, com $61 \%$ dos avaliados categorizados como de alto risco.

REVISTA BRASILEIRA DE EDUCAÇÃO MÉDICA

3 44 (2) : e060; 2020 


\begin{tabular}{|c|c|c|c|c|c|c|c|c|c|c|c|}
\hline \multicolumn{12}{|c|}{ Tabela 2} \\
\hline & & \multicolumn{2}{|c|}{$\begin{array}{l}\text { Com burnout } \\
(n=113)\end{array}$} & \multicolumn{2}{|c|}{$\begin{array}{l}\text { Sem burnout } \\
\qquad(n=21)\end{array}$} & \multicolumn{2}{|c|}{ Total $(n=134)$} & \multicolumn{4}{|c|}{ Regressão logística bivariada } \\
\hline & & \multirow{2}{*}{$\mathbf{N}$} & \multirow{2}{*}{$\%$} & \multirow{2}{*}{$\mathbf{N}$} & \multirow{2}{*}{$\%$} & \multirow{2}{*}{$\mathbf{N}$} & \multirow{2}{*}{$\%$} & \multirow{2}{*}{$\begin{array}{l}\text { Razão de } \\
\text { chance }\end{array}$} & \multirow{2}{*}{ P-valor ${ }^{*}$} & \multicolumn{2}{|c|}{ IC95\% } \\
\hline & & & & & & & & & & LI & LS \\
\hline \multirow{2}{*}{ Sexo } & Feminino & 100 & $88,5 \%$ & 20 & $95,2 \%$ & 120 & $89,6 \%$ & - & - & - & - \\
\hline & Masculino & 13 & $11,5 \%$ & 1 & $4,8 \%$ & 14 & $10,4 \%$ & 2,60 & 0,370 & 0,32 & 21,01 \\
\hline \multirow{2}{*}{ Estado civil } & Solteiro ou divorciado & 93 & $82,3 \%$ & 16 & $76,2 \%$ & 109 & $81,3 \%$ & - & - & - & - \\
\hline & Casado & 19 & $16,8 \%$ & 5 & $23,8 \%$ & 24 & $17,9 \%$ & 0,64 & 0,445 & 0,21 & 1,97 \\
\hline \multirow{2}{*}{ Trabalhava antes } & Não & 84 & $74,3 \%$ & 12 & $57,1 \%$ & 96 & $71,6 \%$ & - & - & - & - \\
\hline & Sim & 29 & $25,7 \%$ & 9 & $42,9 \%$ & 38 & $28,4 \%$ & 0,46 & 0,108 & 0,16 & 1,38 \\
\hline \multirow{2}{*}{ Mudou de cidade } & Não & 76 & $67,3 \%$ & 16 & $76,2 \%$ & 92 & $68,7 \%$ & - & - & - & - \\
\hline & Sim & 37 & $32,7 \%$ & 5 & $23,8 \%$ & 42 & $31,3 \%$ & 1,55 & 0,418 & 0,49 & 5,84 \\
\hline \multirow{2}{*}{ Mora sozinho } & Não & 84 & $74,3 \%$ & 17 & $81,0 \%$ & 101 & $75,4 \%$ & - & - & - & - \\
\hline & Sim & 29 & $25,7 \%$ & 4 & $19,0 \%$ & 33 & $24,6 \%$ & 1,46 & 0,518 & 0,42 & 6,46 \\
\hline \multirow{2}{*}{ Tem filhos } & Não & 103 & $91,2 \%$ & 18 & $85,7 \%$ & 121 & $90,3 \%$ & - & - & - & - \\
\hline & Sim & 10 & $8,8 \%$ & 3 & $14,3 \%$ & 13 & $9,7 \%$ & 0,58 & 0,439 & 0,13 & 3,62 \\
\hline \multirow{2}{*}{ Faz outro curso } & Não & 90 & $79,6 \%$ & 20 & $95,2 \%$ & 110 & $82,1 \%$ & - & - & - & - \\
\hline & Sim & 23 & $20,4 \%$ & 1 & $4,8 \%$ & 24 & $17,9 \%$ & 5,11 & 0,080 & 0,73 & 22,88 \\
\hline \multirow{3}{*}{$\begin{array}{l}\text { Gosta do que faz } \\
\text { nas horas vagas }\end{array}$} & Não & 39 & $34,5 \%$ & 4 & $19,0 \%$ & 43 & $32,1 \%$ & - & - & - & - \\
\hline & Às vezes & 20 & $17,7 \%$ & 5 & $23,8 \%$ & 25 & $18,7 \%$ & 0,41 & 0,209 & 0,07 & 2,16 \\
\hline & Sim & 54 & $47,8 \%$ & 12 & $57,1 \%$ & 66 & $49,3 \%$ & 0,46 & 0,200 & 0,10 & 1,68 \\
\hline \multirow{2}{*}{ Atividade física } & Não & 60 & $53,1 \%$ & 12 & $57,1 \%$ & 72 & $53,7 \%$ & - & - & - & - \\
\hline & Sim & 53 & $46,9 \%$ & 9 & $42,9 \%$ & 62 & $46,3 \%$ & 1,17 & 0,732 & 0,41 & 3,43 \\
\hline \multirow{2}{*}{ Tem religião } & Não & 15 & $13,3 \%$ & 3 & $14,3 \%$ & 18 & $13,4 \%$ & & & & \\
\hline & Sim & 98 & $86,7 \%$ & 18 & $85,7 \%$ & 116 & $86,6 \%$ & 1,08 & 0,900 & 0,18 & 4,44 \\
\hline \multirow{2}{*}{$\begin{array}{c}\text { Processo } \\
\text { terapêutico }\end{array}$} & Não & 103 & $91,2 \%$ & 16 & $76,2 \%$ & 119 & $88,8 \%$ & & & & \\
\hline & Sim & 10 & $8,8 \%$ & 5 & $23,8 \%$ & 15 & $11,2 \%$ & 0,31 & 0,040 & 0,08 & 1,32 \\
\hline
\end{tabular}

*Tabela com associação das variáveis sociodemográficas com o burnout, de acordo com a regressão logística.

$\mathrm{Na}$ avaliação da RP, o escore médio foi de 13,67+-3,99, com 57,5\% em situação de médio risco.

$\mathrm{Na}$ avaliação das COP, o escore atingido foi de 16,92 +- 4,83, e, nas CON, o escore foi de $18,76+-5,52$, demostrando alto risco de desenvolvimento da síndrome de burnout relacionado à falta de bemestar, qualidade de vida e presença de fatores desencadeantes.

\section{DISCUSSÃO}

Em termos gerais, concluiu-se pela alta frequência para síndrome de burnout no grupo pesquisado, com escores elevados para EE, DE e DES. $\mathrm{Na}$ dimensão RP, os escores atingidos demonstram um risco menor.

A frequência encontrada no presente estudo de $84,33 \%$ para síndrome de burnout é superior ao observado por Katsurayama et al. ${ }^{7}$. Em uma avaliação de 107 residentes médicos, observou-se risco moderado ou alto em $70 \%$ dos participantes em consequência das atribuições da residência, seu foco no cuidado do paciente em paralelo com as obrigações institucionais.
O ritmo de trabalho extenuante, talvez pela carga horaria obrigatória ${ }^{8}$, pode ser a causa da EE. Nascimento Sobrinho, Barros, Tironi e Marques Filho ${ }^{9}$ observaram que, entre os 291 médicos plantonistas de uma unidade de terapia intensiva (UTI) em Salvador, $47,6 \%, 24,7 \%$ e $28,4 \%$ pertenciam à categoria de alto risco referente à $\mathrm{EE}$, despersonalização e ineficácia, respectivamente.

Em países que investem na prevenção da síndrome de burnout com debates e ações governamentais, a prevalência da síndrome é mais baixa, variando apenas entre $6 \%$ e $18 \%{ }^{10}$. A implantação de programas de apoio ao profissional pode ser um diferencial na formação dos residentes ${ }^{7}$.

$\mathrm{O}$ alto risco na dimensão DES pode representar uma dificuldade em relacionamentos interpessoais, com comportamentos e atitudes sem interesse ou envolvimento emocional ${ }^{4}$. De acordo com Benevides Pereira ${ }^{5}$, essas características podem ser ainda uma forma de defesa do indivíduo.

A dimensão RP mostrou-se pouco afetada neste estudo, talvez por tratar-se de profissionais no início da carreira, considerando que 71,64\% não trabalhavam antes da residência. 
Fazer outro curso simultâneo foi um fator de risco identificado, sugerindo que esses residentes estariam expostos a estressores em ambos os ambientes de formação, que favorecem a ocorrência da síndrome de burnout. A manutenção de duas atividades simultâneas pode comprometer a saúde e a qualidade de vida dos estudantes, em virtude do desgaste que tais situações originam ${ }^{11}$.

A pesquisa sinaliza que residentes que estão em processo terapêutico têm menos chance de desenvolver burnout, considerando ser um momento em que se consegue ter abertura para se expressar. Uma das formas do tratamento da síndrome de esgotamento profissional envolve, entre outras medidas, a psicoterapia ${ }^{12}$.

Apesar de algumas limitações observadas nesta pesquisa, como a dificuldade de obter um número maior de participantes, vale ressaltar a necessidade de pesquisa qualitativa para melhor definição dos fatores riscos e sobre a proteção contra a síndrome de burnout. Destaca-se ainda a necessidade de promover atividades educativas e de orientação sobre estresse, estratégias de enfrentamento e a divulgação do conhecimento dessa síndrome para a população. Isso permitiria identificar os estressores e as dimensões afetadas, além de estabelecer estratégias mais adequadas para enfrentá-los, pois a prescrição de cada um dos recursos terapêuticos depende da gravidade e da especificidade de cada caso ${ }^{13}$.

\section{CONSIDERAC ÕES FINAIS}

Observa-se alta prevalência da síndrome de burnout nos diversos serviços pesquisados. A resposta ao estresse foi principalmente definida pela presença de exaustão, distanciamento e DES, e a RP se mantém independente do estresse. $O$ fato de fazer outro curso de forma simultânea foi considerado um fator de risco. Aqueles que fazem terapia tiveram uma chance menor de desenvolver a síndrome.

\section{REFERÊNCIAS}

1. Guido LA, Silva RM, Goulart CT, Bolzan MEO, Lopes LFD. Síndrome de burnout em residentes multiprofissionais de uma universidade pública. Rev. Esc. Enferm. USP 2012;46 (6):1477-83 [acesso em 15 set 2018]. Disponível em: http://www.scielo.br/pdf/ reeusp/v46n6/27.pdf.

2. Tarnowski M, Carlotto MS. Síndrome de burnout em estudantes de psicologia. Temas psicol. 2007;15(2):173-80 [acesso em 15 set 2018]. Disponível em: http://pepsic.bvsalud.org/pdf/tp/v15n2/04.pdf.

3. Maslach C, Jackson S. Manual MBI, Inventario "Burnout" de Maslach. Madrid: Publicaciones de Psicología Aplicada; 1997.

4. Galdino MJQ, Martins JT, Haddad MCFL, Robazzi MLCC, Birolim MM. Síndrome de burnout entre mestrandos e doutorandos em enfermagem. Acta paul. enferm. 2016;29(1):100-6 [acesso em 15 set 2018]. Disponível em: http://dx.doi.org/10.1590/1982-0194201600014.

5. Benevides Pereira AMT. Elaboração e validação do ISB Inventário para Avaliação da Síndrome de Burnout. Bol. psicol. 2015;LXV(142):59-71 [acesso em 15 set 2018]. Disponível em: http://pepsic.bvsalud.org/pdf/bolpsi/v65n142/v65n142a06.pdf.

6. Mota ID, Farias GO, Silva R, Folle, A. Síndrome de burnout em estudantes universitários: um olhar sobre as investigações. Motrivivência (Florianópolis) 2017;29:243-56 [acesso em 22 set 2018]. Disponível em: https://periodicos.ufsc.br/index.php/motrivivencia/article/view/21758042.2017v29nespp243. doi:10.5007/2175-8042.2017v29nespp243.

7. Katsurayama M, Gomes MN, Becker MAD, Santos MC, Makimoto FH, Santana LLO. Avaliação dos níveis de estresse psicológico em médicos residentes e não residentes de hospitais universitários. Psicol. hosp. 2011;9(1):75-96 [acesso em maio 2018]. Disponível em: http://pepsic.bvsalud.org/pdf/ph/v9n1/v9n1a05.pdf.

8. Brasil. Presidência da República. Casa Civil. Subchefia para Assuntos Jurídicos. Lei no 11.129, de 30 de junho de 2005. Institui a Residência em Área Profissional de Saúde e cria a Comissão Nacional de Residência Multiprofissional em Saúde (CNRMS).

9. Nascimento Sobrinho CL, Barros DS, Tironi MOS, Marques Filho ES. Médicos de UTI: prevalência da síndrome de burnout, características sociodemográficas e condições de trabalho. Rev. bras. educ. med. 2010;34(1):106-15 [acesso em 13 2018]. Disponível em: http://www. scielo.br/pdf/rbem/v34n1/a13v34n1.pdf.

10. Aronsson G, Theorell T, Grape T, Hammarstrõm A, Marteinsdottr I, Skoog I, et al. A systematic review including meta-analysis of work environment and burnout symptoms. BMC public health 2017;17:1-13 [acesso em 13 out 2018]; 17:264. Disponível em: https://bmcpublichealth. biomedcentral.com/track/pdf/10.1186/s12889-017-4153-7.

11. Silva VLS, Chiquinho NC, Andrade RAPO, Brito MFP, Camelo SHH. Fatores de estresse no último ano do curso de graduação em enfermagem: percepção dos estudantes. Rev. enferm. UERJ 2011;19(1):121-6 [acesso em 15 set 2018]. Disponível em: http:// www.facenf.uerj.br/v19n1/v19n1a20.pdf.

12. Silva LC, Salles TLA. O estresse ocupacional e as formas alternativas de tratamento. Recape - Revista de Carreira e Pessoas 2016;6(2):23447 [acesso em 8 set 2018]. Disponível em: http://revistas.pucsp.br/ $\mathrm{ReCaPe} /$ article/view/29361/20473.

13. Curiel-García JA, Rodríguez-Morán M, Guerrero-Romero F. Síndrome de agotamiento profesional en personal de atención a la salud. Rev. Méd. Inst. Mex. Seguro Soc. 2006;44(3):221-6 [acesso em 22 set 2018]. Disponível em: https://www.medigraphic.com/pdfs/ imss/im-2006/im063e.pdf.

\section{CONTRIBUIÇÃO DOS AUTORES}

Todos os autores participaram da concepção do trabalho, análise de dados, revisão bibliográfica e redação do manuscrito.

\section{CONFLITO DE INTERESSES}

Os autores declaram não haver conflito de interesses neste estudo.

\section{ENDERECO PARA CORRESPONDÊNCIA}

Alessandra Vitorino Naghettini

Rua 235 s/n- setor Leste Universitário, Goiânia- Go, 74605-05

E-mail: alessandra_naghettini@ufg.br 